\title{
Research Square \\ Impact of the COVID-19 Pandemic on the Conduct of Clinical Trials: A Quantitative Analysis
}

Wojciech Margas ( $\nabla$ Wojciech.Margas@creativ-ceutical.com )

Creativ-Ceutical, Poland https://orcid.org/0000-0002-9936-0932

\section{Piotr Wojciechowski}

Creativ-Ceutical, Kraków, Poland

Mondher Toumi

Creativ-Ceutical, Paris, France

\section{Research}

Keywords: clinical trial, COVID-19, forecasting methods, pandemics, regression analysis, time series model

Posted Date: July 15th, 2021

DOl: https://doi.org/10.21203/rs.3.rs-695840/v1

License: @ (i) This work is licensed under a Creative Commons Attribution 4.0 International License. Read Full License 


\section{Abstract}

Background: Globally, healthcare has shouldered much of the socioeconomic brunt of the COVID-19 pandemic. In the area of clinical research, reports indicate that numerous clinical trials have had to be suspended or discontinued. However, these reports have been either qualitative in nature or limited in quantitative scope. The aim of the present analysis was to quantitatively estimate the impact of COVID19 on the number of clinical trials worldwide.

Methods: Data deposited by 219 countries in the ClinicalTrials.gov database between 2007 and 2020 were interrogated using targeted queries. A time series model was fitted to the data for studies ongoing, initiated, or ended between 2007 Quarter (Q) 1 and 2019 Q4 to predict the expected number of trials in 2020 in the absence of COVID-19. The predicted values were compared with the actual 2020 data to quantify the impact of the pandemic.

Results: The number of ongoing registered trials showed continuous growth from 2007 Q1 to 2019 Q4 (from 33,739 to 80,319 trials). By contrast, there were markedly fewer ongoing trials in all four quarters of 2020 compared with forecasted values $(1.6 \%-2.8 \%$ decrease). When excluding COVID-19-related studies, this disparity grew further $(3.4 \%-5.8 \%$ decrease), to a peak of almost 5,000 fewer ongoing trials than estimated for 2020 Q2. Promisingly, however, the number of newly initiated non-COVID-19 trials was higher than predicted in 2020 Q4 (by 9.9\%).

Conclusions: This quantitative analysis confirmed that the pandemic has had an appreciable impact on clinical trials. Provided that current trends persist, clinical trial activities may soon recover to at least preCOVID-19 levels.

\section{Introduction}

The COVID-19 pandemic has had devastating consequences on nearly all societies and economies worldwide (1). Healthcare systems are at the forefront of fighting COVID-19 and have had to bear much of this burden. Since clinical trial research and healthcare are highly interconnected, one would expect the current pandemic to have had a similarly marked impact on clinical trial programs. Indeed, there have been several reports on the unprecedented challenges associated with factors such as patient accrual $(2-4)$, follow-up and data collection $(4,5)$, protocol adherence $(4,5)$, logistics $(6)$, and resource allocation (7), which have resulted in the temporary suspension or even permanent discontinuation of many clinical research and clinical trial programs $(2-4,8,9)$.

To date, the impact of the COVID-19 pandemic on clinical trials has been described in either a qualitative manner (i.e., the identification of problems without quantitative measures) or with a limited quantitative scope. For example, previous reports have focused on individual centers or selected geographic regions (10-12), surveys or case studies that were inherently limited in participant numbers $(11,13,14)$, specific disease areas $(10,11)(12)$, and/or a relatively recent time span $(10),(15,16)$. 
In September 2007, the U.S. Food and Drug Administration Amendments Act made clinical trial registration with the ClinicalTrials.gov database (CTDB) a statutory requirement (17). The CTDB has since undergone steady expansion, and it is currently one of the largest clinical trial databases in the world (18). Now comprising studies from 220 countries, this database has greatly exceeded its initial aim of serving the U.S. market (19). This renders the CTDB a vital source of information for the study of worldwide trends in the development of new therapeutic technologies.

The aim of the present analysis was to quantitatively estimate the global impact of the COVID-19 pandemic on clinical trial programs based on the actual number of trials registered in the CTDB between 2007 and 2020 compared with predicted 2020 values forecasted from pre-COVID-19 data.

\section{Methods}

\subsection{Data source}

All analyses were performed based on information stored in the ClinicalTrials.gov database (19) and the recommendations released by the Clinical Trials Transformation Initiative (20). The studies table is a CTDB key table holding basic information about each registered study; any study-related information provided in other tables can be directly accessed via the studies primary key (the national clinical trial [NCT] number) that uniquely identifies each study. Additional information on the medical subject headings (MeSH) hierarchy used to group the clinical trials into specific health domains was obtained from the mesh_terms table retrieved from the CTDB by remote SQL query (at the time of analysis, this table was present in the CTDB but could not be obtained from the monthly SQL pipe-delimited CTDB snapshot).

\subsection{Definition of outcome variables}

The numbers of clinical trials that were ongoing, started, or ended between 2007 and 2020 were aggregated into quarterly data using the values for the date variables ('start_date', 'study_first_submitted_date', 'completion_date') and the auxiliary information about study status from the 'overall_status' variable. All the information needed for study aggregation was included in the studies dataset.

A total of 852 trials were identified in the studies table as being associated with a clinical device that has not been approved or cleared by the U.S. Food and Drug Administration. These trials were excluded from the analysis, as under U.S. legislation, details regarding these studies are not made publicly available.

Studies containing any of the following prespecified terms in the study keywords, title, summary, description, conditions, interventions, groups, results, or participant data were labeled as COVID-19-related studies: 'COVID-19', 'Coronavirus Disease 2019', 'SARS-CoV-2', ‘SARSr-CoV', 'COVID19', 'NCoV-2019', 'severe acute respiratory syndrome coronavirus 2', '2019-nCoV', '2019 novel coronavirus', and 'Wuhan coronavirus'. 
Using the study keywords, the identified studies were then assigned with at least one of the 4,031 unique MeSH terms (21) grouped into $56 \mathrm{MeSH}$ top-level categories corresponding to the highest levels of the $\mathrm{MeSH}$ tree structure. Among these, $20 \mathrm{MeSH}$ categories comprise health conditions or disease domains, which, in this analysis, have been referred to using the same term: "health domains". Each study could have one or more MeSH terms assigned, thus studies could potentially be classified as falling within more than one health domain. The remaining $36 \mathrm{MeSH}$ categories concerned areas other than health domains and were not used in this analysis. For 62,369 studies (17\%), no MeSH term could be assigned; thus, these studies could not be classified into any of the top-level MeSH categories.

\subsection{Statistical analysis}

Visual inspection of the data indicated a long-term trend of increasing study numbers with each consecutive quarter. Thus, time series analyses were performed using an ETS model to quantitatively estimate the impact of the COVID-19 pandemic on the number of clinical trials being undertaken. More specifically, an ETS model implementation from the CRAN fable library (22) (23) was used, which allowed for automatic model fitting based on the time series trend and seasonality, along with flexible additive, multiplicative, or damped trend components. The model structure was selected from potential candidates based on the Akaike's Information Criterion corrected for small sample bias (AICc), and the Bayesian Information Criterion (BIC). Both criteria consist of a term related to goodness-of-fit and a penalty for complexity, thus allowing a trade-off between model fit and parsimony (24).

The ETS model was fitted to data for the quarterly aggregated number of trials before the outbreak of the pandemic, covering CTDB records for studies ongoing, started, or ended between 2007 quarter (Q) 1 and 2019 Q4. This model was used to predict what the expected number of trials would have been during 2020 Q1-Q4 in the absence of the pandemic. The impact of the COVID-19 pandemic on the number of clinical trials in 2020 was then assessed by comparing the number of trials predicted by the time series model with the actual number of trials observed based on the CTDB records.

Separate forecasts were generated for studies ongoing, started, or ended, as well as for all studies overall and those grouped by health domain. Absolute and relative differences between the actual numbers of studies estimated from the CTDB records and the number of studies predicted by the ETS model were calculated for all studies and the non-COVID-19 subset of studies. Since the COVID-19 pandemic is a recent phenomenon, a forecast for the subset of COVID-19 studies was not feasible because of a lack of data that could be used to fit the ETS model; instead, the number of COVID-19 studies was based on a count of the studies classified as COVID-19-related (as described in Sect. 2.2), and the proportion of COVID-19-related studies was calculated relative to the total number of studies registered in the CTDB.

All analyses were performed using the R software environment for statistical computing (25).

\section{Results}

\subsection{Overall characteristics of registered trials}


In this analysis, the CTDB was interrogated to assess the impact of the COVID-19 pandemic outbreak on the dynamics of clinical trials during 2020. The analysis included 361,706 clinical trials registered in CTDB from the beginning of 2007 until the end of 2020, of which 4,842 (1.3\%) were classified as COVID19-related studies according to the criteria described in the Methods. All other trials were classified as 'non-COVID-19 studies' ( $n=356,864)$. Since the COVID-19 studies were all relatively new, $79.5 \%$ of these trials had the status of 'recruiting', 'not yet recruiting', or 'active, not recruiting', compared with $24.5 \%$ of non-COVID-19 studies with a similar status. There were also marked differences between COVID-19related and unrelated trials regarding the proportion of observational studies (including patient registries) contained within these subgroups (COVID-19: 40\%; non-COVID-19: 21\%) and the main intervention types included (diagnostic tests: $8.7 \%$ and $1.9 \%$; biologics: $10 \%$ and $5.6 \%$; and nonbiologic drugs: $29.8 \%$ and $41.1 \%$ for COVID-19 and non-COVID-19 studies, respectively; see Table 1). Interestingly, when comparing the proportions of intervention types included in the non-COVID-19 and COVID-19 trials, there appears to have been a shift toward biologics in the COVID-19 studies (from 5.6-10\%) at the expense of nonbiologic drugs (from 41.1-29.8\%). The characteristics of the studies analyzed in this paper are summarized in Table 1. 
Table 1

Characteristics of studies registered in the ClinicalTrials.gov database between 2007 and 2020

\section{Non-COVID-19 studies, $\mathrm{n} \quad$ COVID-19 studies, $\mathrm{n} \quad$ Total, $\mathrm{N}(\%)$}

(\%)

$\mathrm{N}$

$17345(4.9 \%)$

$153(0 \%)$

$221(0.1 \%)$

$193902(54.3 \%)$

Completed

Enrolling by invitation

No longer available

Not yet recruiting

Recruiting

Suspended

Temporarily unavailable

Terminated

Unknown last known status:

Active not recruiting

Enrolling by invitation

Not yet recruiting

Recruiting

Withdrawn

Phase

Early Phase 1

Phase 1

Phase 1/Phase 2

$9337(2.6 \%)$

$3403(1 \%)$

$33710(9.4 \%)$

$11363(3.2 \%)$
356864

(\%)

4842

$404(8.3 \%)$

$2(0 \%)$

$24(0.5 \%)$

$641(13.2 \%)$

$163(3.4 \%)$

$5(0.1 \%)$

$1015(21 \%)$

2429 (50.2\%)

$27(0.6 \%)$

0 (0\%)

$53(1.1 \%)$

$0(0 \%)$

$0(0 \%)$

0 (0\%)

0 (0\%)

79 (1.6\%)

42 (0.9\%)

3445 (1\%)
206 (4.3\%)

154 (3.2\%)
(53.8\%)
17749 (4.9\%)

155 (0\%)

$245(0.1 \%)$

194543

3393 (0.9\%)

$296(0.1 \%)$

17856 (4.9\%)

55565 (15.4\%)

1829 (0.5\%)

19 (0\%)

20525 (5.7\%)

7706 (2.1\%)

1587 (0.4\%)

8051 (2.2\%)

22771 (6.3\%)

$9416(2.6 \%)$

${ }^{a}$ Not available.

bStudies were classified based on intervention type when at least one study arm listed the specific category. 


\begin{tabular}{|c|c|c|c|}
\hline & $\begin{array}{l}\text { Non-COVID-19 studies, n } \\
\text { (\%) }\end{array}$ & $\begin{array}{l}\text { COVID-19 studies, } n \\
\text { (\%) }\end{array}$ & Total, N (\%) \\
\hline Phase 2 & $46507(13 \%)$ & 607 (12.5\%) & $47114(13 \%)$ \\
\hline Phase 2/Phase 3 & $5424(1.5 \%)$ & $173(3.6 \%)$ & $5597(1.5 \%)$ \\
\hline Phase 3 & $32169(9 \%)$ & $407(8.4 \%)$ & $32576(9 \%)$ \\
\hline Phase 4 & $27464(7.7 \%)$ & $131(2.7 \%)$ & 27595 (7.6\%) \\
\hline Not applicable & $120360(33.7 \%)$ & $1144(23.6 \%)$ & $\begin{array}{l}121504 \\
(33.6 \%)\end{array}$ \\
\hline$N A^{a}$ & $76464(21.4 \%)$ & 1978 (40.9\%) & $78442(21.7 \%)$ \\
\hline \multicolumn{4}{|l|}{ Type } \\
\hline Expanded Access & $684(0.2 \%)$ & $31(0.6 \%)$ & $715(0.2 \%)$ \\
\hline Interventional & $280402(78.6 \%)$ & $2864(59.1 \%)$ & $\begin{array}{l}283266 \\
(78.3 \%)\end{array}$ \\
\hline Observational & 69017 (19.3\%) & 1681 (34.7\%) & 70698 (19.5\%) \\
\hline $\begin{array}{l}\text { Observational [Patient } \\
\text { Registry] }\end{array}$ & $6761(1.9 \%)$ & $266(5.5 \%)$ & 7027 (1.9\%) \\
\hline \multicolumn{4}{|l|}{ Intervention type } \\
\hline Behavioral & $33564(9.4 \%)$ & 477 (9.9\%) & 34041 (9.4\%) \\
\hline Biologic & $20060(5.6 \%)$ & $486(10 \%)$ & $20546(5.7 \%)$ \\
\hline Combination Product & $1121(0.3 \%)$ & $41(0.8 \%)$ & $1162(0.3 \%)$ \\
\hline Device & $42805(12 \%)$ & $282(5.8 \%)$ & 43087 (11.9\%) \\
\hline Diagnostic Test & $6774(1.9 \%)$ & $421(8.7 \%)$ & $7195(2 \%)$ \\
\hline Dietary Supplement & $10647(3 \%)$ & $104(2.1 \%)$ & $10751(3 \%)$ \\
\hline Nonbiologic Drug & $146718(41.1 \%)$ & $1444(29.8 \%)$ & $148162(41 \%)$ \\
\hline Gene Therapy & $1897(0.5 \%)$ & $13(0.3 \%)$ & $1910(0.5 \%)$ \\
\hline Procedure & $35684(10 \%)$ & $129(2.7 \%)$ & $35813(9.9 \%)$ \\
\hline Radiation & $7327(2.1 \%)$ & $25(0.5 \%)$ & $7352(2 \%)$ \\
\hline Other & $63195(17.7 \%)$ & $1327(27.4 \%)$ & $64522(17.8 \%)$ \\
\hline \multicolumn{4}{|l|}{ 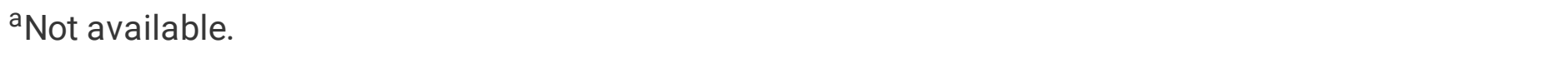 } \\
\hline $\begin{array}{l}\text { bStudies were classifi } \\
\text { category. }\end{array}$ & intervention $\mathrm{t}$ & st one s & he specific \\
\hline
\end{tabular}




\begin{tabular}{|c|c|c|c|}
\hline & $\begin{array}{l}\text { Non-COVID-19 studies, } n \\
(\%)\end{array}$ & $\begin{array}{l}\text { COVID-19 studies, } n \\
(\%)\end{array}$ & Total, N (\%) \\
\hline$N A^{a}$ & $36616(10.3 \%)$ & 709 (14.6\%) & $37325(10.3 \%)$ \\
\hline a Not available. & & & \\
\hline
\end{tabular}

\subsection{Trends in clinical trials in the pre-COVID-19 and COVID- 19 eras}

Continuous growth was observed in the number of registered clinical trials with 'ongoing' status from 33,739 trials at the beginning of 2007 to 80,319 trials at the end of 2019 (Fig. 1). The year 2020 clearly deviated from this long-term trend: for the first time during the period analyzed, the number of ongoing trials was lower compared with that of the previous year. This drop spanned all quarters of 2020, with the first two quarters being the most affected. There were 631 fewer ongoing studies in 2020 Q1 compared with 2019 Q1, and 1,099 fewer ongoing studies in 2020 Q2 compared with 2019 Q2.

When comparing the actual numbers of ongoing clinical trials with the numbers forecasted by the ETS model, the estimated number of "missing" ongoing trials was $-1,890,-2,286,-1,557$, and $-1,311$ for each of the consecutive four quarters of 2020 , which corresponded to a $1.6-2.8 \%$ decrease vs. the predicted values (Fig. 2). This disparity further increased to 5.4-5.8\% between 2020 Q2 and Q4 when COVID-19-related studies were excluded (see also Additional file 1).

Analysis of the quarterly trends in 2020 indicated that the observed decreases in the number of ongoing clinical trials during this year were mostly attributable to the lower-than-expected number of studies initiated in Q1 (-13.6\%) and Q2 (- 10.5\%) and the higher-than-expected termination rate in Q1 (7.7\%) and Q4 (41.3\%) (Fig. 2). Further, the number of non-COVID-19 studies initiated in Q1 and Q2 of 2020 dropped by $20.4 \%$ and $38.2 \%$, respectively, relative to the forecasted values, and this drop was not fully compensated for by the upsurge in new COVID-19 studies. In the second half of 2020, an excess of initiated studies was observed over predictions, which would have helped to mitigate the lower number of ongoing studies observed during the first half of the year. Lastly, 2020 Q4 presented a markedly higher proportion of terminated trials than predicted $(41 \%)$, by contrast with the relatively small disparities between the forecasted and actual number of trials ended during 2020 Q1-Q3.

\subsection{Trends in clinical trials across different health domains}

Of the 361,706 trials identified during 2007-2020, 299,337 (82.8\%) trials could be assigned to $\geq 1$ of the 20 health domains according to their associated MeSH terms. The ETS model was applied to each of these health domains to evaluate the impact of the pandemic on clinical trials within specific areas of therapeutic research. Detailed results for all 20 health domains are presented in Additional file 2. 


\subsubsection{Trends in clinical trials on infectious diseases}

Comparisons of the predicted versus actual numbers of clinical trials indicated that the area of infectious diseases encompassed the health domains most affected by the COVID-19 pandemic, namely viral diseases, respiratory tract diseases, and bacterial infections and mycoses (Fig. 3). These 3 health domains shared a distinct trend in the number of ongoing trials in 2020 that was opposite to that observed for clinical trials overall, in that the observed number of ongoing trials in these domains during 2020 Q2-Q4 was larger than the predicted values.

This disparity between the predicted and actual number of trials was greatest in the domain of viral diseases, reaching 21.0-24.2\% more ongoing trials than the forecasted values during 2020 Q2-Q4 (Fig. 3). This dynamic was associated with a staggering increase in the number of new studies initiated throughout 2020, reaching 234\% and 98\% more new studies than forecasted for Q2 and Q3, respectively. The domain of respiratory tract diseases was the second-most impacted, peaking at $7.2 \%$ more ongoing studies (Q3) and 74\% more new studies (Q2) than forecasted for 2020. Lastly, the bacterial infections and mycoses health domain had an initial $2.7 \%$ decrease in the number of ongoing studies in Q1, followed by an increase to a peak of 3.5\% in Q3. The number of new studies in this domain jumped from 4.5-48.3\% above the forecast in the first half of 2020 and stabilized to almost $30 \%$ above the forecast during the last two quarters of the year.

When excluding all COVID-19-related studies from the analysis, the numbers of ongoing and initiated studies were lower in 2020 than predicted (Fig. 3), which suggests that studies initiated in response to the COVID-19 outbreak (see Additional file 3) were responsible for the observed increases in the total number of ongoing and initiated trials on infectious diseases during 2020.

\subsubsection{Trends in clinical trials on noninfectious diseases}

By contrast with the area of infectious disease, decreasing trends were observed for most of 2020 in the total numbers of ongoing and initiated trials in domains related to noncommunicable diseases. These domains included cardiovascular diseases, neoplasms, mental disorders, and diseases of the musculoskeletal and nervous systems (Fig. 4). The numbers of ongoing trials in these 5 domains remained below the forecasted values throughout 2020, and this trend was associated with a marked decrease in the number of studies initiated within the first half of 2020. Notably, however, the number of studies initiated in 2020 Q4 within these domains exceeded the forecasts (indeed, this was the case for all 20 health domains; see Additional file 2). This increase compensated at least in part for the Q1-Q3 reduction in ongoing trials within the domains associated with cardiovascular diseases, neoplasms, and mental disorders. As expected, the disparities between the predicted and actual number of trials overall versus that of the non-COVID-19 subset of studies were much smaller than those observed for the infectious disease domains.

\section{Discussion}


This report describes the application of a well-established time series model to ClinicalTrials.gov registry data to quantitatively estimate the effect of the COVID-19 pandemic on the number of clinical trials in 2020. The approach described here is unique, given that 13 years of pre-COVID-19 clinical trial registry data were included in the model (previous analyses have relied on more recent data, with the earliest data being from 2015 (26)). This facilitated the observation of both seasonal and long-term trends, and it further allowed robust forecasts of what the dynamics might have been in the absence of COVID-19.

The COVID-19 pandemic is an unprecedented event that continues to leave its mark on all areas of socioeconomic functioning. This analysis has confirmed that the pandemic led to the widespread suspension of ongoing trials and postponement of planned studies. Based on the ETS model, the number of ongoing trials in 2020 Q2 would have been higher by approximately 2,300 trials if the pandemic had not occurred. This gap decreased later during the year, partly because of an increase in new studies related to COVID-19, but the number of ongoing trials in 2020 Q4 remained lower than expected by approximately 1,300 trials. If studies related to COVID-19 are excluded, this gap increases to a peak of almost 5,000 in 2020 Q2.

Besides the pronounced drop in clinical trial numbers in 2020, there was also a slight but noticeable decrease in 2019 when considering the long-term trend observed over the period of 2007-2018. The reasons for this are unclear, but this decrease might have been related to 2019 being the year of the lowest world economic growth since the financial crisis of 2008-2009 (27). This makes 2019 a poor reference for year-to-year comparisons, as it would lead to underestimation of the impact of COVID-19 on the dynamics of clinical trials during 2020. To overcome the limitations of such an approach, we based our analysis on a comparison of the actual number of trials in 2020 Q1-Q4 in the CTDB with those forecasted by an ETS model fitted to data from the entire available historical pre-COVID-19 timespan (2007-2019) (23).

The field of clinical research has shown great flexibility and the ability to accommodate completely new conditions. This was illustrated by the upsurge in new experimental trials related to COVID-19 that were registered in Q2-relatively soon after the outbreak began. One consequence of which, was that the number of ongoing trials in the area of infectious diseases noticeably exceeded predictions based on data from previous years. This implies that less than a quarter was required to design, prepare, and initiate these new clinical trials. Secondly, a re-emergence of non-COVID-19 trials was observed in the second half of 2020, such that the number of trials registered in 2020 Q4 exceeded the predictions for all 20 of the health domains. Lastly, the observed shift from traditional drugs to biologics in COVID-19 studies may reflect the recent advancements in bioinformatics and molecular biology that have accelerated the development pathway for new therapies (including vaccines) compared with the traditional research approaches applied to small molecules (28).

Despite the increase in new trials in 2020 Q4, the overall number of ongoing studies at the end of 2020 remained lower than expected based on trends over the pre-COVID-19 era. However, looking at the recent upward trend in new trials, there is a chance that the expected level of active trials will soon be attained. 
This is of paramount importance to the pharmaceutical industry, clinicians, and patients, since drugs undergoing clinical trials have the potential to increase the spectrum of available therapies for a variety of diseases, and any delays in clinical trials may translate into a loss of clinical benefit (29). The higherthan-forecasted proportions of trials ended in 2020 Q4 might have also been associated with the financial closure of projects at the end of the year, due to the postponement of difficult financial decisions regarding trial closure to the end of the annual financial cycle (for many companies, this coincides with the end of the calendar year).

Several risk mitigation measures have been implemented to reduce the likelihood of SARS-CoV-2 transmission and lessen the impact of the pandemic on clinical trial programs $(3,4,10,11,14,30,31)$. These measures have included staggering staff shifts, prioritizing specific trials and canceling or postponing others, pausing patient recruitment for ongoing trials, substituting in-person site monitoring visits and patient visits with technology-based solutions wherever feasible (e.g., using e-consent methods, telemedicine consults, remote electronic medical record access, and virtual monitoring of data), extending patient study visit windows, and shipping oral drugs directly to patients. Regulatory agencies such as the U.S. Food and Drug Administration (32) and the European Medicines Agency (33), as well as various research, philanthropic, and advocacy organizations, have issued guidance to help address the challenges inherent in conducting clinical trials in the pandemic era (11).

Although the pandemic has been disruptive to clinical programs worldwide, experts believe that many of the adaptations made to mitigate this impact and rapidly generate high-quality evidence in COVID-19related trials are beneficial outside of this context, offering long-term opportunities to transform the clinical trial landscape $(4,9,11,34-37)$. Several of these improvements are considered long overdue even in the absence of COVID-19, such as more streamlined trial designs and the widespread adoption of digital and remote technologies.

The approach used in this study has several limitations. First, not all clinical trials are registered in the CTDB. Second, since all studies with keywords related to COVID-19 were classified as COVID-19-related, it is possible that the proportion of COVID-19 trials was overestimated by including studies amended, suspended, terminated, or withdrawn for any reason related to the COVID-19 outbreak. To estimate the extent of this overestimation, we re-ran the analysis with COVID-19-related studies redefined as only those with COVID-19-related keywords in the study title. We found that $84 \%$ of studies with a keyword related to COVID-19 in any field also had such a keyword in the title; these studies were, therefore, highly likely to actually comprise COVID-19-related trials. Thus, we concluded that the approach used to classify studies in this analysis provided a good trade-off between sensitivity and specificity and did not lead to substantial overestimation of the proportion of studies related to COVID-19 therapies. Third, this analysis was based on a "snapshot" of the data published by the CTDB on January 1,2021, and this dataset might not have contained updates of the CTDB records from 2020 that were delayed. Fourth, although study records submitted to the CTDB are subject to quality control and the Posting Procedures guidelines (38), users have substantial freedom to enter customized records. Thus, inconsistencies, inaccuracies, and deficiencies in the entered information (including dates) are unavoidable. 


\section{Conclusions}

This report describes the application of an established forecasting model to global clinical trial data from 2007 to 2020 to quantitatively evaluate the impact of the COVID-19 pandemic. Although the findings confirm that COVID-19 has had an appreciable impact, the data suggest that clinical trial activities may soon recover to at least pre-COVID-19 levels if current trends persist. Although the negative effect of COVID-19 on clinical trials appears to be lessening, it remains important for study sponsors, contract research organizations, and regulatory agencies to continue to monitor this highly dynamic situation.

\section{Abbreviations}

AICc, Akaike's Information Criterion corrected for small sample bias

BIC, Bayesian Information Criterion

COVID-19, Coronavirus Disease 2019

CTDB, ClinicalTrials.gov Database

$\mathrm{MeSH}$, medical subject headings

Q, quarter

SARS-CoV-2, severe acute respiratory syndrome coronavirus 2

\section{Declarations}

\section{Ethics approval and consent to participate}

Not applicable.

\section{Consent for publication}

Not applicable.

\section{Availability of data and materials}

The original database snapshot supporting the conclusions of this article is available from the Clinical Trials Transformation Initiative (https://aact.ctti-clinicaltrials.org/pipe_files). The specific database snapshots and/or analysis outcomes used are available from the corresponding author on reasonable request. The custom codes developed and used for the analyses are not freely available as they are the property of Creativ-Ceutical and intended for internal use only.

\section{Competing interests}


All authors are employees of Creativ-Ceutical.

\section{Funding}

This study was funded by Creativ-Ceutical SARL (Brasseur, Luxembourg).

\section{Authors' contributions}

WM and PW compiled the registry data and performed the time series analyses. WM, PW, and MT interpreted the results and drafted the manuscript. All authors read and approved the final manuscript.

\section{Acknowledgments}

The authors thank Samuel Aballéa (Creativ-Ceutical, Rotterdam, Netherlands) and Sylvaine Barbier (Creativ-Ceutical, Lyon, France) for their review of a draft of this manuscript and their valuable comments. Medical writing support was provided by Natasha Beeton-Kempen of ApotheCom (London, UK) and was funded by Creativ-Ceutical SARL (Brasseur, Luxembourg).

\section{References}

1. United Nations Department of Economic and Social Affairs. World economic situation and prospects 2021. https://www.un.org/development/desa/dpad/publication/world-economic-situation-andprospects-2021/. Accessed 19 May 2021.

2. Deroose CM, Lecouvet FE, Collette L, Oprea-Lager DE, Kunz WG, Bidaut L, et al. Impact of the COVID19 crisis on imaging in oncological trials. Eur J Nucl Med Mol Imaging. 2020;47:2054-8.

3. Caruso C. Clinical research slows as COVID-19 surges. Cancer Discov. 2020;10(5):630.

4. Upadhaya S, Yu JX, Oliva C, Hooton M, Hodge J, Hubbard-Lucey VM. Impact of COVID-19 on oncology clinical trials. Nat Rev Drug Discov. 2020;19(6):376-7.

5. Bagiella E, Bhatt DL, Gaudino M. The consequences of the COVID-19 pandemic on non-COVID-19 clinical trials. J Am Coll Cardiol. 2020;76(3):342-5.

6. Chowdhury P, Paul SK, Kaisar S, Moktadir MA. COVID-19 pandemic related supply chain studies: A systematic review. Transp Res E Logist Transp Rev. 2021;148:102271.

7. Wieten S, Burgart A, Cho M. Resource allocation in COVID-19 research: Which trials? Which patients? Am J Bioeth. 2020;20(7):86-8.

8. Carlisle BG. Clinical trials stopped by Covid-19 https://covid19.bgcarlisle.com/. Accessed 19 May 2021.

9. van Dorn A. COVID-19 and readjusting clinical trials. Lancet. 2020;396(10250):523-4.

10. Rodriguez-Otero P, Reis J, Alfonso-Pierola A, Salas-Benito D, Giraldez M, Azanza JR, et al. Singleinstitution experience in clinical trials during the COVID-19 pandemic in Spain: Not so bad after all? JCO Glob Oncol. 2020;6:904-5. 
11. Waterhouse DM, Harvey RD, Hurley P, Levit LA, Kim ES, Klepin HD, et al. Early impact of COVID-19 on the conduct of oncology clinical trials and long-term opportunities for transformation: Findings from an American Society of Clinical Oncology survey. JCO Oncol Pract. 2020;16(7):417-21.

12. Strujo $E$, Sanders $M$, Fiscella $K$, Thomas $M$, Johnson B, Deets A, et al. COVID-19 impact on multi-site recruitment and enrollment. Clin Trials. 2020;17(5):501-4.

13. Kent DG, Knapp DJHF, Kannan N. Survey says: "COVID-19 lockdown hits young faculty and clinical trials". Stem Cell Reports. 2020;15(1):1-5.

14. Medidata Solutions Inc. The impact of COVID-19 on clinical trial sites. April 2020. https://www.medidata.com/wp-content/uploads/2020/05/COVID19-Site-Survey_20200518_v1.pdf. Accessed 19 May 2021.

15. Lee J, Shin HW, Lee JY, Kim JS, Yang JW, Lee KH, et al. A comprehensive analysis of clinical trials in the COVID-19 pandemic era. Medicina (Kaunas). 2020;56(6):315.

16. Gaudino M, Arvind V, Hameed I, Di Franco A, Spadaccio C, Bhatt DL, et al. Effects of the COVID-19 pandemic on active non-COVID clinical trials. J Am Coll Cardiol. 2020;76(13):1605-6.

17. Food and Drug Administration. Food and Drug Administration Amendment Act of 2007. https://www.govinfo.gov/content/pkg/PLAW-110publ85/html/PLAW-110publ85.htm. Accessed 19 May 2021.

18. Stergiopolous S, Getz K, Blazynski C. Evaluating the completeness of ClinicalTrials.gov. Ther Innov Regul Sci. 2019;53(3):307-17.

19. Gillen JE, Tse T, Ide NC, McCray A. Design, implementation and management of a web-based data entry system for ClinicalTrials.gov. Stud Health Technol Inform. 2004;107:1466-70.

20. Perez RP, Finnigan S, Patel K, Whitney S, Forrest A. Clinical trial electronic portals for expedited safety reporting: Recommendations from the Clinical Trials Transformation Initiative Investigational New Drug Safety Advancement Project. JMIR Cancer. 2016;2(2):e16.

21. U.S. National Library of Medicine. Medical Subject Headings 2021. https://meshb.nlm.nih.gov/search. Accessed 19 May 2021.

22. O'Hara-Wild M, Hyndman R, Wang E, Caceres G, Hensel T-G, Hyndman T. fable: Forecasting models for tidy time series. 2021. https://cran.r-project.org/web/packages/fable/index.html. Accessed 19 May 2021.

23. Hyndman RJ, Athanasopoulos G. Forecasting: Principles and practice. 3rd ed. Melbourne, Australia: OTexts; 2021. Available from: ttps://otexts.com/fpp3/. Accessed 19 May 2021.

24. Dziak JJ, Coffman DL, Lanza ST, Li R, Jermiin LS. Sensitivity and specificity of information criteria. Brief Bioinform. 2020;21(2):553-65.

25. R Core Team. R: A language and environment for statistical computing Vienna, Austria: R Foundation for Statistical Computing; 2021. Available from: https://www.R-project.org/. Accessed 19 May 2021.

26. Unger JM, Xiao H. The COVID-19 pandemic and new clinical trial activations. Trials. 2021;22(1):260. 
27. United Nations Department of Economic and Social Affairs. World economic situation and prospects 2020. https://unctad.org/system/files/official-document/wesp2020_en.pdf. Accessed 19 May 2021.

28. Hufsky F, Lamkiewicz K, Almeida A, Aouacheria A, Arighi C, Bateman A, et al. Computational strategies to combat COVID-19: useful tools to accelerate SARS-CoV-2 and coronavirus research. Brief Bioinform. 2021;22(2):642-63.

29. Byrd JB, Bello N, Meyer MN. Pandemic pandemonium: Pausing clinical research during the COVID-19 outbreak. Circulation. 2020;141(25):2045-7.

30. Goldsack JC, Izmailova ES, Menetski JP, Hoffmann SC, Groenen PMA, Wagner JA. Remote digital monitoring in clinical trials in the time of COVID-19. Nat Rev Drug Discov. 2020;19(6):378-9.

31. McDermott MM, Newman AB. Preserving clinical trial integrity during the coronavirus pandemic. JAMA. 2020;323(21):2135-6.

32. Food and Drug Administration. Conduct of clinical trials of medical products during the COVID-19 public health emergency 2020 [updated January 2021]. https://www.fda.gov/regulatoryinformation/search-fda-guidance-documents/fda-guidance-conduct-clinical-trials-medical-productsduring-covid-19-public-health-emergency. Accessed 19 May 2021.

33. European Medicines Agency. Guidance on the management of clinical trials during the COVID-19 (coronavirus) pandemic 2020 [updated February 2021].

https://www.ema.europa.eu/en/news/guidance-sponsors-how-manage-clinical-trials-during-covid19-pandemic. Accessed 19 May 2021.

34. Xue JZ, Smietana K, Poda P, Webster K, Yang G, Agrawal G. Clinical trial recovery from COVID-19 disruption. Nat Rev Drug Discov. 2020;19(10):662-3.

35. Gaba P, Bhatt DL. The COVID-19 pandemic: a catalyst to improve clinical trials. Nat Rev Cardiol. 2020;17(11):673-5.

36. MacKenzie R, Honig P, Sewards J, Goodwin R, Hellio M-P. COVID-19 must catalyse changes to clinical development. Nat Rev Drug Discov. 2020;19(10):653-4.

37. Mitchell EJ, Ahmed K, Breeman S, Cotton S, Constable L, Ferry G, et al. It is unprecedented: trial management during the COVID-19 pandemic and beyond. Trials. 2020;21(1):784.

38. ClinicalTrials.gov. Updated quality control and posting procedures 2019. https://prsinfo.clinicaltrials.gov/webinars/Webinar_Posting_Summary_Results_within_30_days.pdf. Accessed 19 May 2021.

\section{Figures}




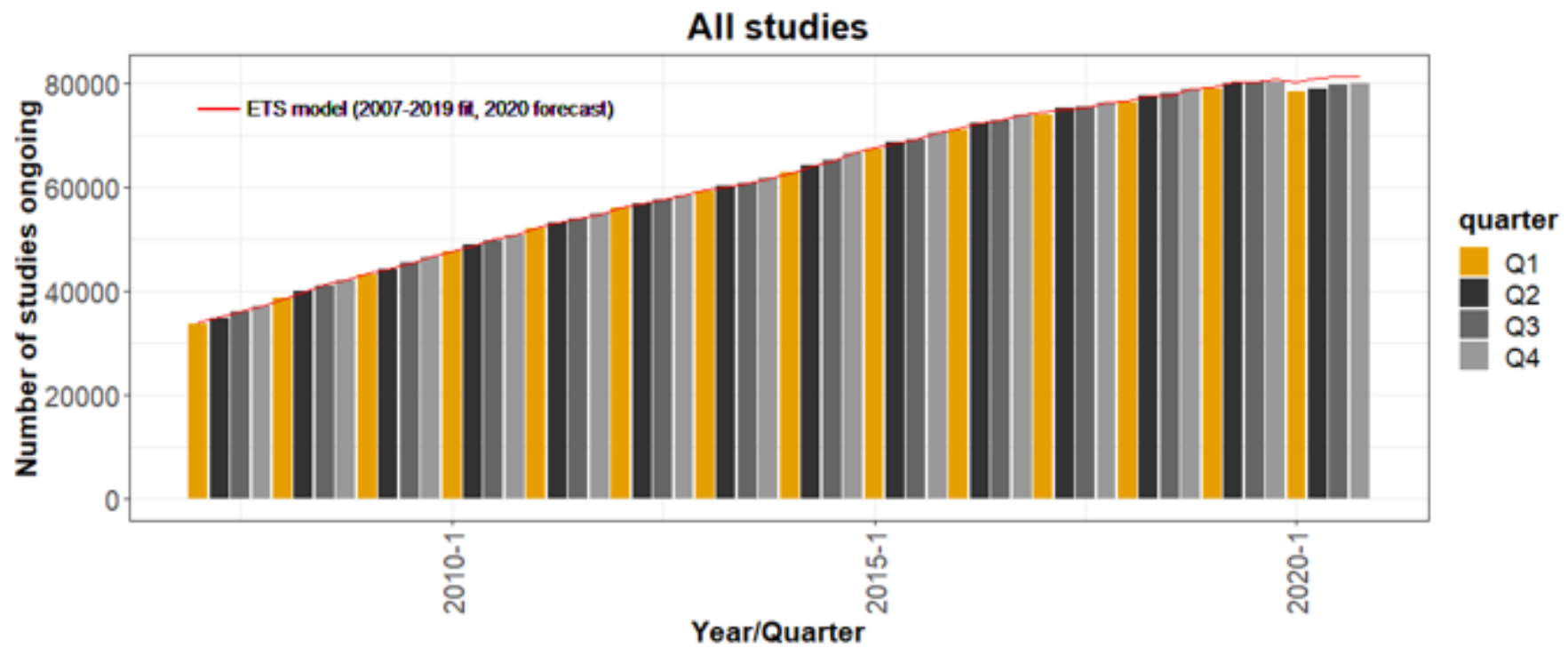

Figure 1

Dynamics of the number of ongoing trials between 2007 and 2020. The red line represents the ETS model fitted to data from the ClinicalTrials.gov database for the number of ongoing clinical trials during the preCOVID-19 period (2007-2019), and the forecasted numbers for the four quarters of 2020 predicted by the ETS model.

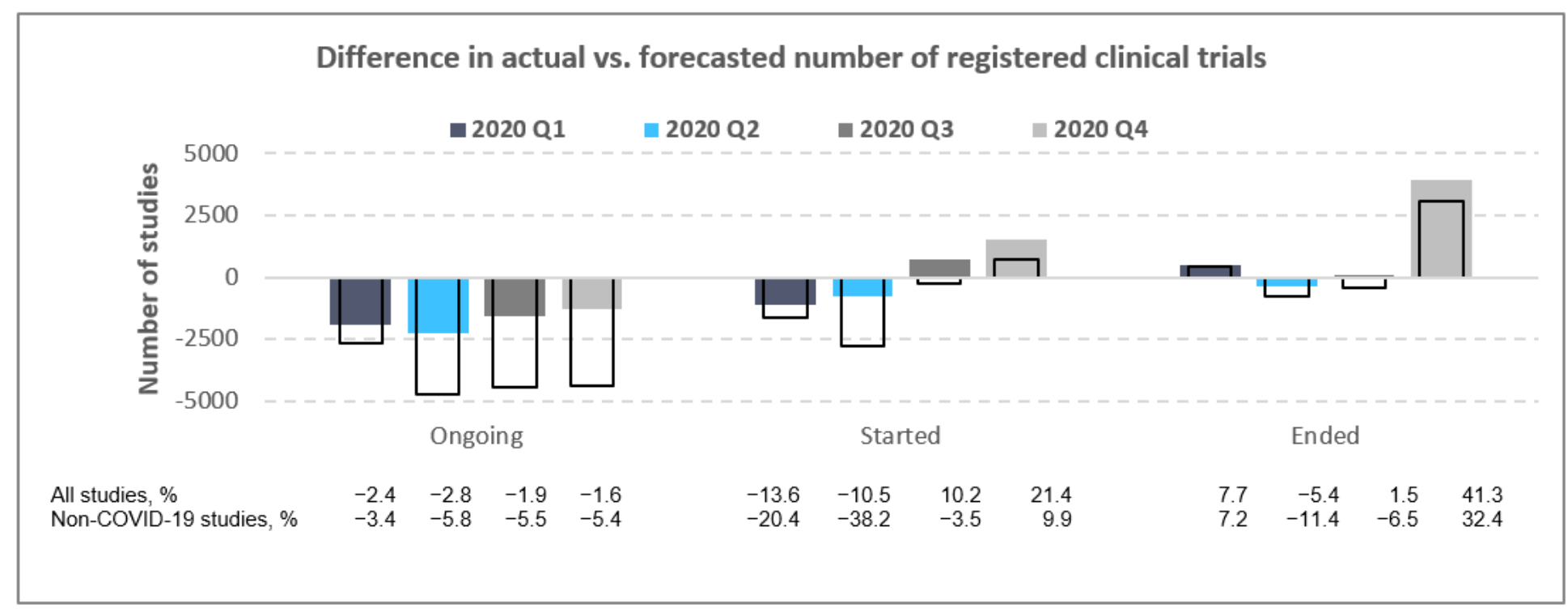

Figure 2

Differences between the actual and predicted number of clinical trials in 2020 Q1-Q4. Values represent the difference between the actual numbers of registered clinical trials in 2020 and the forecasted values based on the ETS model fitted to data from 2007 to 2019. Colored bars, all studies; empty bars, nonCOVID-19 studies only. The proportions (\%) in the bottom panel were calculated relative to the number of predicted studies for each quarter. 


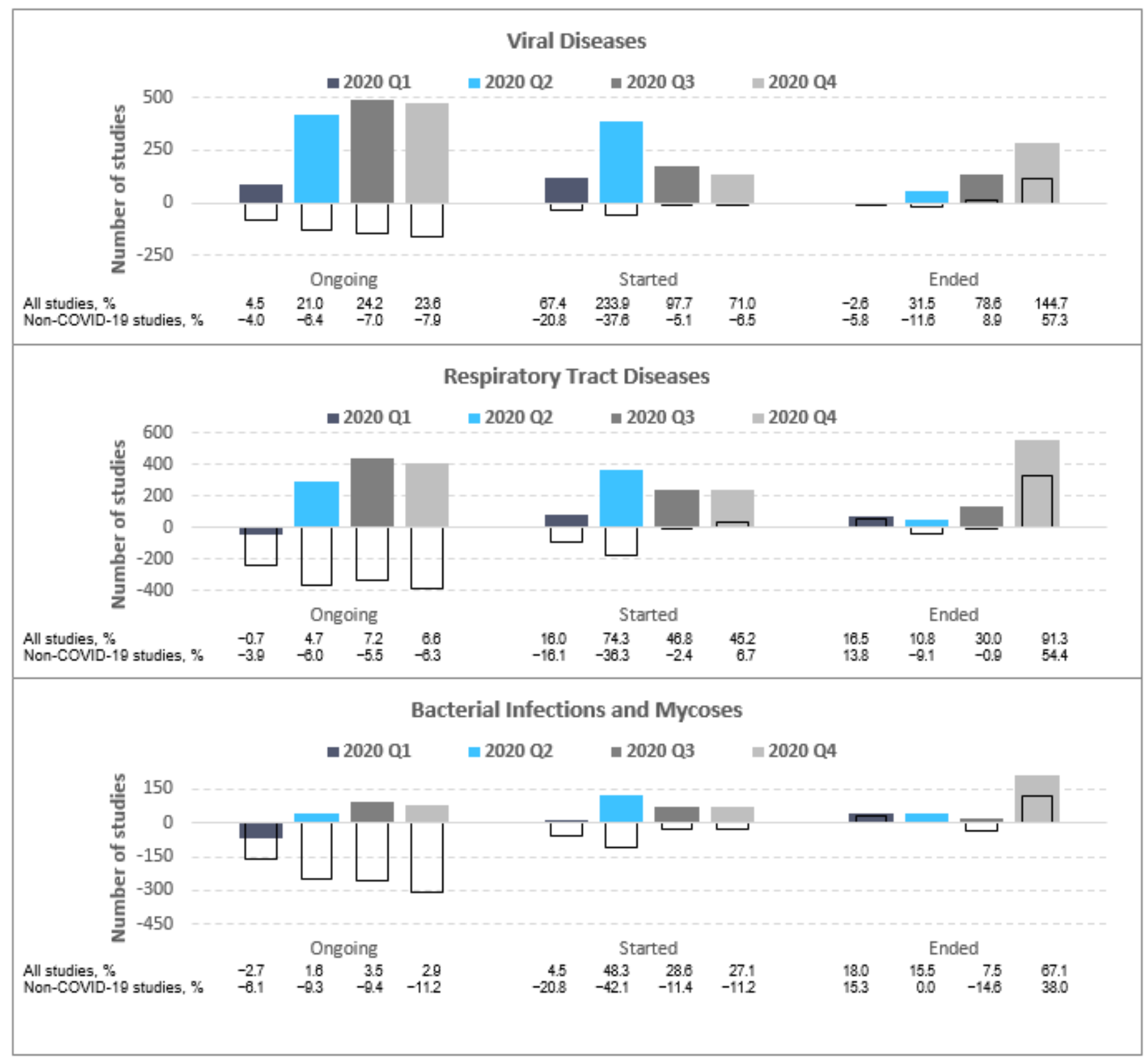

Figure 3

Differences between the actual and predicted number of clinical trials on infectious diseases. Values represent the difference between the actual numbers of registered clinical trials in 2020 and the forecasted values based on the ETS model fitted to data from 2007 to 2019. Colored bars, all studies; empty bars, non-COVID-19 studies only. The proportions (\%) in the bottom panel were calculated relative to the number of predicted studies for each quarter. 


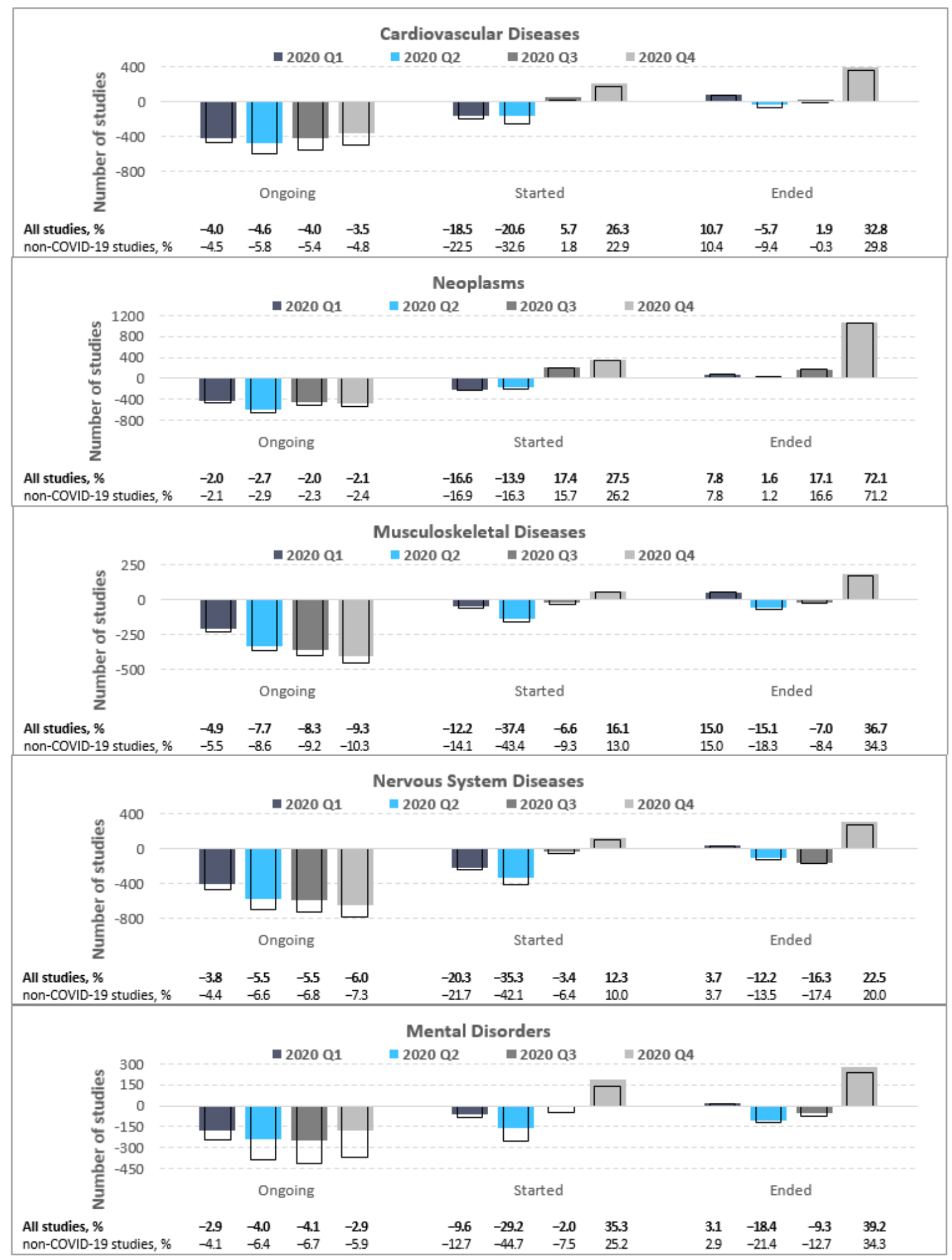

\section{Figure 4}

Differences between the actual and predicted number of clinical trials on noncommunicable diseases. Values represent the difference between the actual numbers of registered clinical trials in 2020 and the forecasted values based on the ETS model fitted to data from 2007 to 2019. Colored bars, all studies; empty bars, non-COVID-19 studies only. The proportions (\%) in the bottom panel were calculated relative to the number of predicted studies for each quarter. 


\section{Supplementary Files}

This is a list of supplementary files associated with this preprint. Click to download.

- SQUIRE2.0checklist.pdf

- Additionalfile1revised27May21.docx

- Additionalfile2revised27May21.docx

- Additionalfile3revised27May21.docx 